\title{
Purification and Nature of the Antibiotic Nisin
}

\author{
By N. J. BERRIDGE \\ National Institute for Research in Dairying, University of Reading \\ AND G. G. F. NEWTON AND E. P. ABRAHAM \\ Sir William Dunn School of Pathology, University of Oxford
}

(Received 12 February 1952)

The production of nisin by a strain of Streptococcus lactis (Lancefield group $\mathrm{N}$ ) was described by Mattick \& Hirsch (1944, 1947). Berridge (1949) obtained the antibiotic in purified form by fractional precipitation with salt and 'crystallization' from $80 \%(\mathrm{v} / \mathrm{v})$ ethanol. This product was found to yield alanine, valine, leucine and isoleucine, and possibly cystine and aspartic acid, on hydrolysis. A solubility curve, however, showed that it contained at least two substances, the less soluble of which amounted to about two-thirds of the whole.

Hirsch (1951) studied the relative antibacterial activity of three batches of nisin under various conditions and concluded that he was dealing with several antibiotics. Berridge (1952) investigated the behaviour of several preparations of nisin on counter-current distribution in twenty tubes. $\mathrm{He}$ obtained evidence that some of the preparations contained two active components, although the resolution was not sufficient to permit a confident analysis of the curve. Bavin, Beach, Falconer \& Friedmann (1952) stated that a counter-current distribution curve of nisin showed two active peaks.

The aim of the work described in the present paper was to resolve a single batch of nisin into its components, and to isolate these components in quantities sufficient for some of their biological and chemical properties to be studied. The antibacterial properties of the components obtained are described by Gowans, Smith \& Florey (1952).

\section{RESOLUTION OF NISIN INTO SEVERAL SIMILAR POLYPEPTIDES}

\section{Methods}

Solvent system. The resolution was carried out in a single experiment by counter-current distribution in a 102-tube all-glass apparatus (Craig, Hausmann, Ahrens \& Harfenist, 1951). The starting material was a preparation of nisin purified by Bengers Ltd. (batch 706 P). Preliminary experiments showed that nisin had an overall partition coefficient close to unity in a solvent system consisting of methanol, $n$-butanol and acetate buffer at $\mathrm{pH}$ 3.0. Although nisin was not very soluble in it, this system was used for two reasons. First, because the starting material distributed between the two phases in approximately equal concentrations: other things being equal the maximum resolving power is obtained from a limited number of transfers when the geometric mean of the partition coefficients of the components of a mixture approaches unity (Craig \& Craig, 1950). Secondly, because the $\mathrm{pH}$ of the system was one at which some groups in the material appeared to be partly ionized: differences in solubility due to differences in the dissociation constants of ionizable groups may occur under such conditions. A system in which nisin was more soluble could readily have been obtained by lowering the $\mathrm{pH}$, but it would not have had these two advantages. Moreover, nisin was particularly stable in the solvent used. A number of solutions which were allowed to stand at room temperature for a month showed no loss in antibacterial activity.

Estimation of material in different fractions. The low solubility of nisin in the solvent system made it impracticable to determine the amount of material in the various fractions by weighing. The amount was determined in two ways: (1) by measuring total $\mathrm{N}$ by the micro-Kjeldahl procedure, and (2) by measuring the colour obtained when the mixture of amino-acids liberated on hydrolysis was treated with ninydrin in the manner described by Moore \& Stein (1948). Reasonable agreement was found between the two sets of determinations.

Activities were measured by the turbidimetric method of Berridge \& Barrett (1951).

Procedure. The 102 tubes of the machine were arranged in two rows of 51, one above the other, and a recycling device was fitted between tube 101 and tube 0 to enable the solutes to make a second run through the apparatus (Craig et al. 1951). The starting material was loaded equally into the first five tubes of the apparatus, and the fundamental procedure used until the leading upper layer was adjacent to tube 0 . At this stage a single withdrawal of lower layers from the trailing edge was begun. Fresh lower layers were placed in the tubes thus left empty and the leading upper layers were recycled over them. By this procedure the nisin was subjected to 150 transfers.

Analysis of distribution curves. Values for the partition coefficients of the main components were obtained by examining successive points along the distribution curve.

Williamson \& Craig (1947) showed that after $n$ transfers have been made with a single substance placed initially in tube 0 the relationship between the fraction $T_{r}$ of the substance in tube $r$, the fraction $T_{r+1}$ in tube $r+1$, and the partition coefficient $K$, is given by

$$
\frac{T_{r}}{T_{r+1}}=F^{\prime}\left[\frac{1}{K}\right], \text { where } \quad F^{\prime}=\frac{(r+1)}{(n-r)} .
$$


When more than one tube is loaded at the beginning of the distribution this expression needs modification. Suppose that the number of tubes loaded is $x$. The final distribution
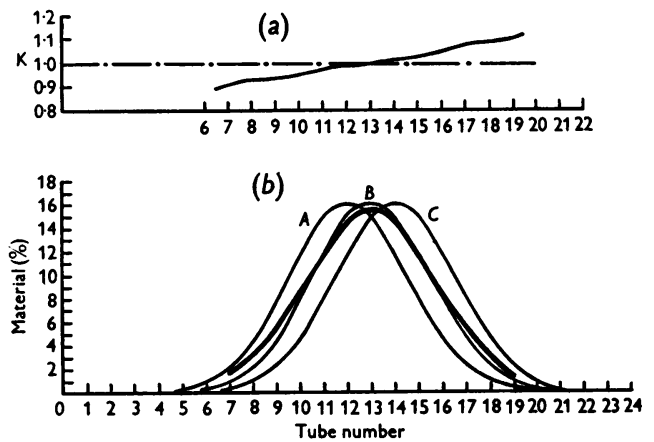

Fig. 1. (a) Values of $K$ found from equation 2 (see text). (b) Effect of loading a substance with $K=1$ into the first three tubes before a 24-transfer distribution. Thin-lined curves $A, B$ and $C$ : distribution of the material initially in tubes 0,1 and 2 , respectively. Thick-lined curve: distribution of the total material which would be found experimentally.

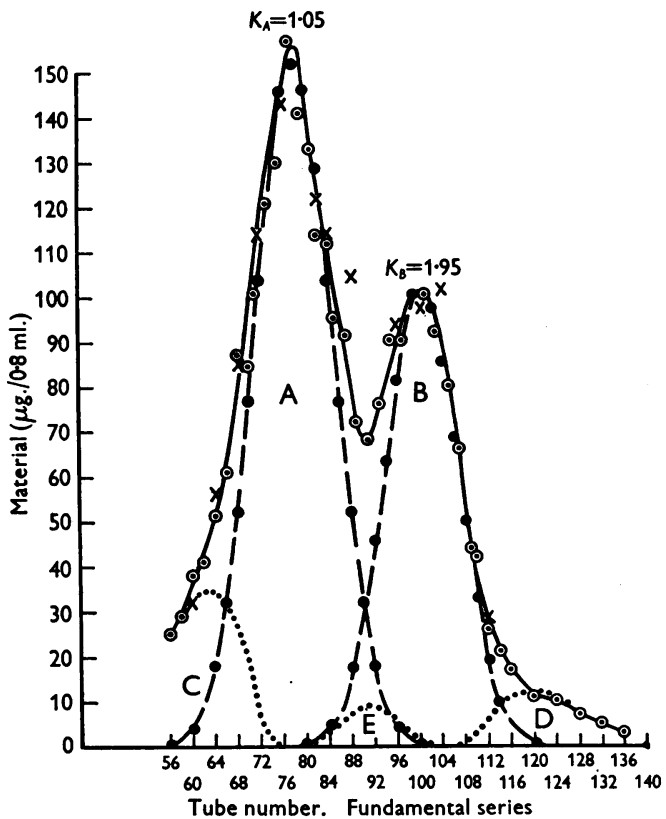

Fig. 2. 150-transfer distribution of nisin (preparation 706 P) in a solvent system consisting of acetic acid, sodium

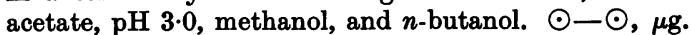
material $/ 0.8 \mathrm{ml}$. of mixed layers estimated colorimetrically; $x-x, \mu \mathrm{g}$. material $/ 0.8 \mathrm{ml}$. of mixed layers, estimated by the micro-Kjeldahl method; --0 , calculated curves; ......, approximate interpretation for remaining material.

curve can then be regarded as the sum of a series of $x$ separate curves, all having the same shape but each advanced one tube in front of its successor. If the separate curves are nearly symmetrical their summation will yield a curve whose maximum is midway between the separate maxima at each end of the series (Fig. 1). This curve cannot be described exactly as a simple function of $n, T_{r}, T_{r+1}$, and $K$, but its maximum will be in the same position as that of the curve derived solely from material loaded into tube $\frac{1}{2}(x-1)$, and if $x$ is small and $n$ large it will fit the latter rather closely.

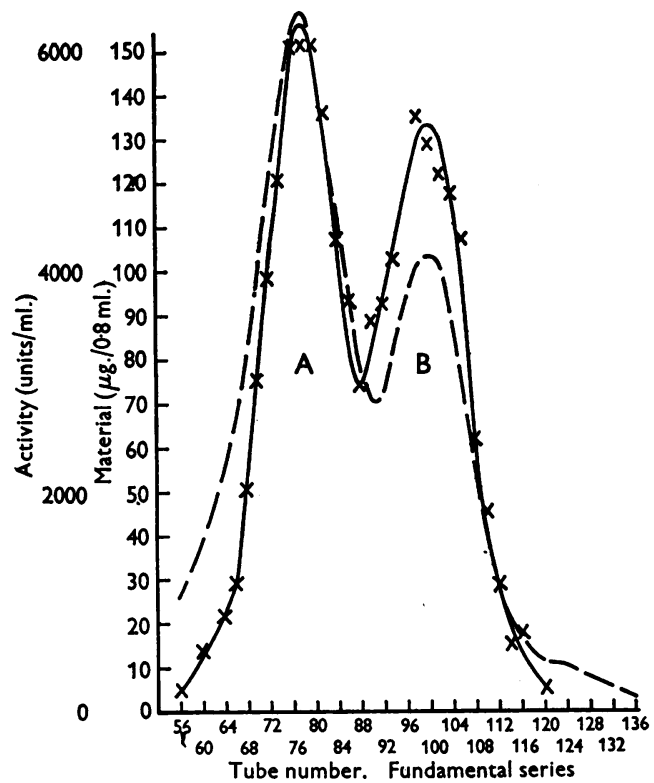

Fig. 3. 150-transfer distribution of nisin $(706 \mathrm{P})$ in a solvent system consisting of acetic acid-sodium acetate, $\mathrm{pH} 3 \cdot 0$, methanol, and $n$-butanol. $\times-\times$, antibacterial activity/ml. determined turbidimetrically; - - curve showing total material from Fig. 2.

The curve derived from material loaded into tube $\frac{1}{2}(x-1)$ is described by the equation

$$
\frac{T_{r}}{T_{r+1}}=F^{\prime}\left[\frac{1}{K}\right], \text { where } \quad F^{\prime}=\frac{\left[r+1-\frac{1}{2}(x-1)\right]}{\left[n-r+\frac{1}{2}(x-1)\right]} .
$$

Equation 2 can therefore be used for finding $K$ when material is loaded into $x$ tubes. If the material is homogeneous, and $x$ is small and $n$ large, the values obtained from different parts of the curve will show only minor variation; but they will be slightly larger on the leading side of the curve than on the trailing side, and will coincide with the true value at the maximum (Fig. 1).

Values of $K$ were determined in this way over what appeared to be homogeneous portions of the curve, and these values were used for constructing sets of five separate theoretical curves each advanced one tube in front of the other. The curve obtained by summation of the separate theoretical curves could be fitted closely to the curve determined by experiment.

\section{RESULTS}

Counter-current distribution. The distribution curve shown in Fig. 2 indicates that the preparation of nisin was a complex mixture containing $49 \% \mathrm{~A}$, 
$32 \% \mathrm{~B}, 12 \% \mathrm{C}, 5 \% \mathrm{D}$ and possibly $3 \%$ of another substance E. Fig. 3 shows the distribution of activity.

Table 1, which should be compared with Fig. 2, shows the fractions that were selected for pooling and reconcentration. The specific activity of $\mathbf{A}$ against the standard test organism was similar to that of $B$ and about five times that of $C$ and $D$. The antibacterial properties of the concentrates were studied further by Gowans et al. (1952).

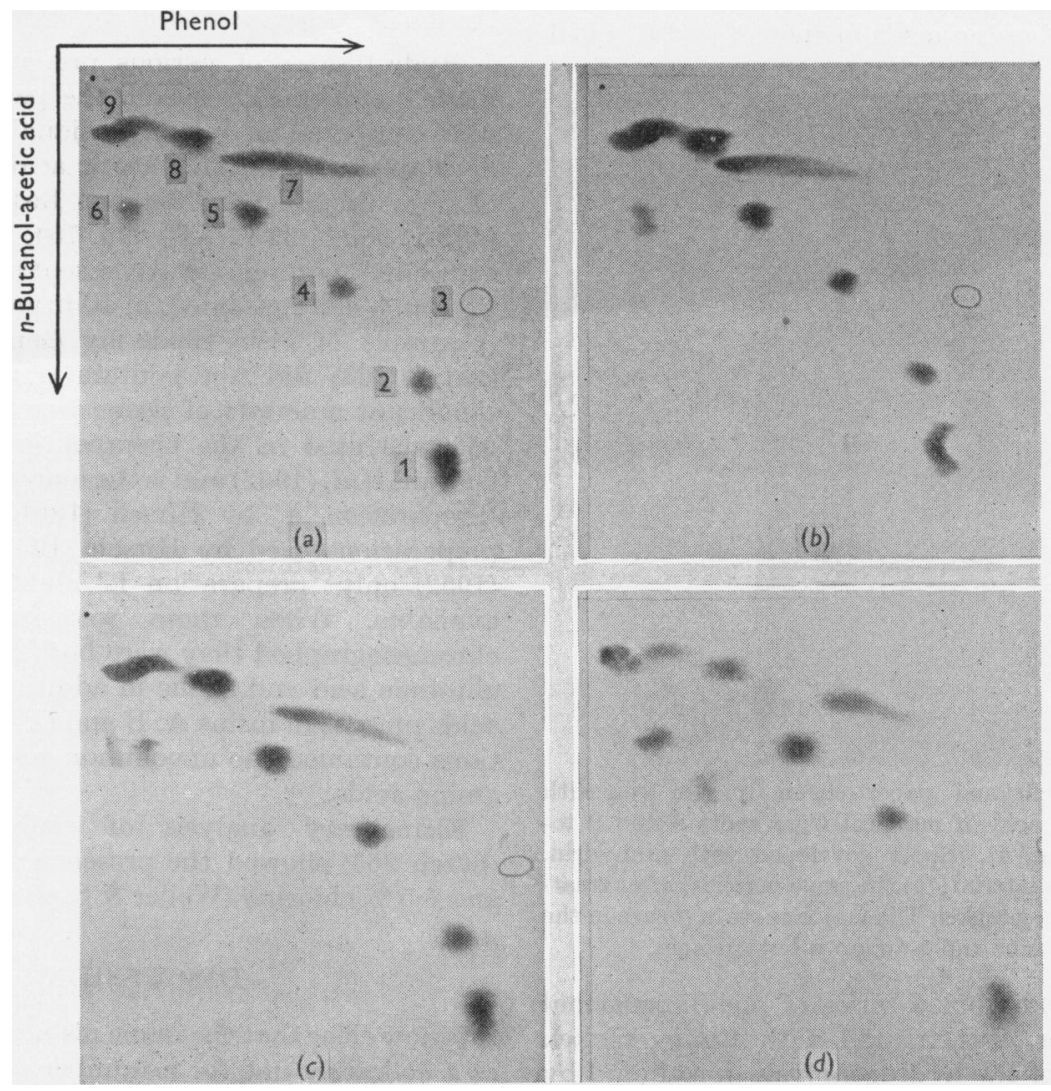

Fig. 4. Two-dimensional paper chromatograms of nisins A, B, C and D. (a), A; (b), B; (c), C; (d), D. Spots developed with ninhdyrin: (1) leucine and/or isoleucine; (2) valine and methionine; (3) proline; (4) alanine; (5) glycine; (6) aspartic acid; (7) lysine and histidine; (8) cystathionine; (9) lanthionine.

Table 1. Antibacterial activities of the concentrates prepared from the pooled fractions obtained after counter-current distribution

$\begin{array}{cccc} & \begin{array}{c}\text { Fundamental } \\ \text { series }\end{array} & \begin{array}{c}\text { Withdrawn } \\ \text { series }\end{array} & \begin{array}{c}\text { Units* of } \\ \text { antibacterial } \\ \text { activity/mg. } \\ \text { against Strep. }\end{array} \\ \text { A } & 76-81 & - & 32000 \\ \text { B } & 102-108 & - & 41500 \\ \text { C } & 56-62 & 54-40 & 10-5000 \\ \text { D } & 116-140 & - & 10-5000 \\ \text { E } & 90-94 & - & \text { Not known } \\ & * \text { The unit is that defined by Berridge (1949). }\end{array}$

\section{AMINO-ACID COMPOSITION OF THE NISIN POLYPEPTIDES}

\author{
Nisins $A, B, C$ and $D$
}

Fig. 4 shows two-dimensional paper chromatograms obtained from acid hydrolysates of nisins A, B, C and $D$. The chromatograms were developed first with butanol-acetic acid (Woiwod, 1949) and sub. 
fact that it showed no colour when treated with Ehrlich's reagent.

The four nisins also contain two substances which react with ninhydrin but which are not normally found in protein hydrolysates. These are shown as spots 8 and 9 in Fig. 4. After treatment of the hydrolysate with hydrogen peroxide they appeared as a double spot close to the origin. Further information about the nature of these substances was obtained by hydrogenolysis. A mixture of the amino-acids in spots 8 and 9 , which

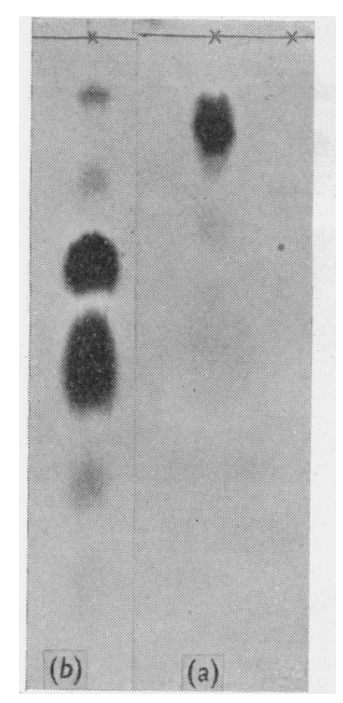

Fig. 5. One-dimensional paper chromatogram run with butanol-acetic acid of material from spots 8 and 9 together (see Fig. 4). Spots developed with ninhydrin. (a) Untreated material; (b) the same material after treatment with Raney nickel. The major spots in $(b)$ are in the positions of alanine and $\alpha$-amino- $n$-butyric acid.

had been eluted from a series of one-dimensional chromatograms, was treated with Raney nickel. The products of the hydrogenolysis were found by paper chromatography to be alanine and $\alpha$-amino$n$-butyric acid in about equal amounts (Fig. 5). Hydrogenolysis of 1 part of lanthionine and 2 parts of cystathionine yielded a mixture which showed the same amino-acid pattern, although it contained a smaller proportion of $\alpha$-amino- $n$-butyric acid. These results indicated that lanthionine and cystathionine or allocystathionine might be present in the mixture.

To simulate a nisin hydrolysate, a mixture was made of the following amino-acids in simple proportions by weight: leucine (4), valine (2), alanine (2), glycine (2), proline (2), serine (1), lysine (2), histidine (2), aspartic acid (1), methionine (2), lanthionine (1), and cystathionine (4).

The pattern formed by this mixture in twodimensional paper chromatograms did in fact reproduce very closely that formed by a nisin hydrolysate, both before and after treatment with hydrogen peroxide. Moreover, no new spots were seen after chromatographing a nisin hydrolysate to which lanthionine and cystathionine had been added.

Nisin D would appear to contain some of the sulphur-containing amino-acids in the oxidized form (Fig. 4).

\section{Amino-acid composition of different batches of nisin}

Hydrolysates of various preparations of nisin, made commercially during the period of 1948-51, were compared on one-dimensional chromatograms developed with butanol-acetic acid. Eight batches of nisin prepared by Bengers Ltd. during 1948-9 (652 A, 660P, 33Y, XP, 696, 754, 706P, EPS 756) contained the amino-acids present in nisins $A, B$ and C. Batch 696 contained, in addition, glutamic acid. A sample of nisin made by Genatosan in 1949 (batch 263) did not contain glutamic acid but significant amounts of serine were present. Batch 263 was used in the therapeutic experiments of Gowans et al. (1952) and is the material referred to as 'preparation A' by Hirsch (1951). Of the other preparations used by Hirsch (1951) and Berridge (1952) only 'preparation $L$ ' and batch 138 were available. When these were hydrolysed and chromatographed they were both found to contain glutamic acid and serine in addition to the aminoacids present in nisins A, B and C. All the preparations contained the uncommon sulphur-containing amino-acids.

Elementary analysis of nisin hydrochloride (batch 263) showed the presence of 5.6\% sulphur and $5.5 \%$ chlorine (Weiler \& Strauss).

\section{DISCUSSION}

It is now clear that the name nisin must be regarded as a collective one for a number of closely related polypeptides. In this respect nisin resembles several other antibiotics obtained from bacteria. For example the gramicidins, tyrocidines (Craig, Gregory \& Barry, 1949), licheniformins (Callow \& Work, 1952) and bacitracins (Newton \& Abraham, 1950,1952 ) occur as families of similar peptides.

The main active polypeptides in the single preparation of nisin that was resolved by countercurrent distribution resembled each other very closely in amino-acid composition. Other batches of nisin that had not been purified by counter-current distribution also contained a similar series of amino-acids, although glutamic acid and serine were present in some preparations but not in others.

The nisin polypeptides are remarkable for their high content of sulphur. In addition to methionine, which is commonly found in proteins, they appear to 
contain lanthionine and a considerable amount of cystathionine.

Lanthionine has been isolated from acid hydrolysates of wool that has been treated with alkali (Horn, Jones \& Ringel, 1941). It is assumed to be formed, on alkali treatment, from cystine residues in the protein, although it has been detected in small amounts in an acid hydrolysate of the tips of virgin wool (Consden, Gordon \& Martin, 1946).

Cystathionine has been isolated from the mycelium of a certain strain of Neurospora and has been shown to be an intermediate in the biosynthesis of methionine (Horowitz, 1947). It appears to have also been obtained from wool treated with sodium sulphide (Küster \& Irion, 1929), but there have been no reports of its occurrence in a native protein.

Although lanthionine occurs rarely, if at all, in proteins, it is present in the antibiotic subtilin, a polypeptide produced by a strain of Bacillus subtilis (Dimick, Alderton, Lewis, Lightbody \& Ferrold, 1947). Lewis \& Alderton (1948) found that subtilin contained $4.8 \%$ sulphur, and that on acid hydrolysis it yielded lanthionine and an amino-acid with the empirical formula $\mathrm{C}_{7} \mathrm{H}_{14} \mathrm{O}_{4} \mathrm{~N}_{2} \mathrm{~S}$. It seems not unlikely that the latter was a cystathionine or allocystathionine.

Nisin and subtilin show some general similarities in their amino-acid composition and also in their physical and antibacterial properties. However, the two substances are clearly distinct. Nisin contains no tryptophan and subtilin no methionine.

\section{EXPERIMENTAL}

\section{Solvents}

'Analar' glacial acetic acid, $n$-butanol of laboratory reagent grade and commercial refined methanol (B.S.S. no. 506 (1950), Imperial Chemical Industries Ltd.) were used without further purification.

\section{Estimation of material}

(a) Micro-Kjeldahl. The procedure of Tompkins \& Kirk (1942) was used, with the digestion mixture of Campbell \& Hanna (1937) and a digestion period of 40-50 min.

(b) Photometric ninhydrin. The method described by Newton \& Abraham (1950) was used. The colour obtained from nisin after hydrolysis was very close to that obtained from an equal weight of leucine. Samples of $2 \mathrm{ml}$. volumes were taken from each layer of the contents of the evennumbered tubes of the fundamental series, and $3 \mathrm{ml}$. samples from the single layer of the withdrawn series. Estimations on the odd-numbered tubes 71-109 inclusive were also made in an independent experiment.

\section{Measurement of antibacterial activity}

The turbidimetric method of Berridge \& Barrett (1951) was used. By using a syringe for making serial dilutions, an automatic pipette for measuring volumes, and a culture of the test organism in its logarithmic phase of growth, this method enabled a large number of assays to be completed in replicate, simultaneously, and in a few hours. Some causes of variation were thereby eliminated and an improvement in accuracy was obtained.

\section{Counter-current distribution}

Solvent system. The mixture was made by adjusting a solution of $400 \mathrm{ml}$. of glacial acetic acid in $3200 \mathrm{ml}$. of water to $\mathrm{pH} 3.0$ with concentrated $\mathrm{NaOH}$, and adding $400 \mathrm{ml}$. of methanol and $2000 \mathrm{ml}$. of $n$-butanol. Such a mixture, after equilibration, gave a $\mathrm{pH}$ reading with the glass electrode of 3.56 for the lower phase and 3.52 for the upper phase. The quantity of $\mathrm{NaOH}$ needed was very low, $\mathrm{pH} \mathrm{3.0}$ being near the beginning of the titration curve of acetic acid. The solvent was kept no longer than overnight to reach equilibrium before use.

Distribution procedure. The fixed volume of the stationary lower layer was $20 \mathrm{ml}$. The machine was filled with solvent, as described by Craig et al. (1951). The contents of tubes 0-4 inclusive were then removed and to each of these tubes were added $60 \mathrm{mg}$. of purified nisin dissolved in a mixture of $20 \mathrm{ml}$. top phase and $20 \mathrm{ml}$. bottom phase. Altogether, $300 \mathrm{mg}$. of nisin were therefore used in the distribution.

The fundamental procedure for operating the machine (Craig \& Post, 1949) was then carried out, using $20 \mathrm{ml}$. quantities of upper layer, until the upper layer which was originally in tube 4 had reached tube 99 . At this stage a fresh upper layer was not put into tube 0 . A further transfer was then made and the contents of tube 0 (a lower layer only) were removed as the first member of the withdrawn series. Tube 0 was left empty. The cycle of operations was repeated and the contents of tube 1 were withdrawn. The 'front' of the mobile phase was now in tube 101, the last tube of the bottom row of the machine. Therefore an excess of lower layer (about $40 \mathrm{ml}$.) was put in tube 0 so as to provide a fresh lower layer for the first step of the recycle. After the next transfer and withdrawal an increment of fresh lower layer was added to tube 1 . This cycle of operations was continued until the 'front' of the upper layers had reached tube 52 for the second time. All the material which was originally put into the machine had now completed 150 transfers.

Concentration of pooled samples. In each case $\mathrm{HCl}$ was added to the mixture until the $\mathrm{pH}$ reading fell to $\mathbf{1 \cdot 9} \pm 0 \cdot 1$, and the solution was then concentrated in vacuo with a bath temperature of $50^{\circ}$. The apparent $\mathrm{pH}$ was maintained between 1.8 and 2.0 by periodic adjustments, from four to six interruptions being necessary during the concentration of each batch.

In the absence of butanol frothing was very troublesome, so that on occasions when all the butanol had evaporated before the end of the concentrating, extra butanol was added when the $\mathrm{pH}$ was adjusted. In this way 10 - to 20 -fold concentration was readily achieved.

To each concentrate were added 40 vol. of acetone (the amount usually used in preparation work is 10 vol.). The resulting precipitate of nisin and $\mathrm{NaCl}$ was collected, by decantation and centrifuging, into a small tube and extracted four times with $1 \mathrm{ml}$. of $0.05 \mathrm{~N}-\mathrm{HCl}$. The $\mathrm{NaCl}$ dissolved first and, as nisin is of low solubility in saturated salt solution, the first extracts were of low activity. The four solutions were therefore bulked according to their assay values. The preparations were left as concentrated solutions in acid, since nisin is quite stable under these conditions, 
and it is difficult to prepare dry powders without the risk of loss, particularly on such a small scale.

Minor variations of the above procedure were tried with less success.

\section{Hydrolysis of nisin}

The sample of nisin was sealed in a hard-glass tube with $6 \mathrm{~N}-\mathrm{HCl}$ (1 ml. of acid was used for a $10 \mathrm{mg}$. sample) and heated at $110^{\circ}$ for $24 \mathrm{hr}$. The hydrolysate was freed from $\mathrm{HCl}$ by evaporation in an air stream at $100^{\circ}$.

\section{Preparation of paper chromatograms}

Whatman no. 1 paper was used, and $400 \mu \mathrm{g}$. of material were applied to the paper for each two-dimensional chromatogram. The chromatograms were developed at $21^{\circ}$ with a butanol-acetic acid-water mixture (Woiwod, 1949). They were then dried in a current of air at $50-70^{\circ}$ until most of the acetic acid had been removed. Phenol-water (4:1), in presence of the vapour of $50 \%$ (w/v) acetic acid was used as the second solvent (Dent, 1948). After the phenol had run, the papers were dried in a stream of warm air at less than $40^{\circ}$. The positions of the amino-acids were revealed by treatment with ninhydrin (Woiwod, 1949).

To oxidize the sulphur-containing amino-acids $30 \%$ $(w / v) \mathrm{H}_{2} \mathrm{O}_{2}$ was mixed with the sample before applying it to the paper (Dent, 1948).

\section{Hydrogenolysis with Raney nickel}

Raney nickel was prepared from nickel-aluminium alloy by the method of Mozingo, Wolf, Harris \& Folkers (1943).

\section{Preparation of $\alpha$-amino-n-butyric acid}

Methionine (200 mg.) was dissolved in water (15 ml.) and one spatula-full of Raney nickel was added to the solution. The mixture was heated under reflux in an oil bath at $120^{\circ}$ for $3 \mathrm{hr}$. The Raney nickel was removed by centrifuging and washed with a small amount of water. Nickel was then removed from the solution by shaking three times with an equal volume of 8-hydroxyquinoline in $\mathrm{CHCl}_{3}$. After shaking three times with an equal volume of $\mathrm{CHCl}_{3}$, to remove traces of 8-hydroxyquinoline, the aqueous solution was evaporated to dryness. The residue $(170 \mathrm{mg}$.) was dissolved in a small amount of hot water, and ethanol added until the solution was just turbid. On cooling, $\alpha$-amino- $n$ butyric acid separated in crystalline form. It was shown by paper chromatography to be homogeneous.

\section{Hydrogenolysis of lanthionine and cystathionine}

A mixture of lanthionine (1 mg.) and cystathionine (1.6 mg.) was dissolved in $5 \mathrm{ml}$. water and a small spatulafull of Raney nickel added to the solution. The mixture was heated under reflux for $3 \mathrm{hr}$., and the Raney nickel removed at the centrifuge. The resulting solution was shaken with 8-hydroxyquinoline in $\mathrm{CHCl}_{3}$ and then with $\mathrm{CHCl}_{3}$ alone. The aqueous solution was evaporated to dryness and the residue chromatographed on paper with butanol-acetic acid.

\section{Hydrogenolysis of spots 9 and 8}

Twenty spots of hydrolysed nisin 263 (500 $\mu \mathrm{g}$. each) were chromatographed in one dimension on a single sheet of paper. The chromatogram was developed with butanolacetic acid (Woiwod, 1949). The paper was freed from solvent and then heated at $115^{\circ}$ for $20 \mathrm{~min}$. The positions occupied by the amino-acids on the paper could then be clearly seen in ultraviolet light. The area occupied by spots 8 and 9 were cut out and the amino-acids eluted from the paper by the method of Sanger \& Tuppy (1951). A small spatula-full of Raney nickel was then added to the eluate and the mixture heated under reflux in an oil bath at $120^{\circ}$ for $3 \mathrm{hr}$. The Raney nickel was removed by centrifuging and washed with a small amount of water. The supernatant was shaken with a solution of 8-hydroxyquinoline in $\mathrm{CHCl}_{3}$ and then three times with $\mathrm{CHCl}_{3}$ alone. The resulting aqueous solution was evaporated to dryness and the residues examined on a onedimensional paper chromatogram developed with butanolacetic acid (Fig. 5).

\section{SUMMARY}

1. A single preparation of nisin has been resolved into at least four active polypeptides by countercurrent distribution between solvents. These substances have been named nisin A, B, C and D.

2. Nisins $A$ and $B$ show similar activity against Streptococcus agalactiae. Nisins $\mathrm{C}$ and $\mathrm{D}$ are about one-fifth as active as $A$ and $B$.

3. Nisins A, B and C appear to contain leucine and/or isoleucine, valine, alanine, glycine, proline, aspartic acid, histidine, lysine, methionine, lanthionine and a cystathionine or allocystathionine. Cystathionine appears to be present in larger amount by weight than most of the other aminoacids. Nisin $\mathrm{D}$ contains glutamic acid but no valine or methionine.

4. Various commercial preparations of nisin have been found to be similar in amino-acid composition, but some batches contain glutamic acid and serine, which are absent from nisins A, B and C.

5. The nisin polypeptides show some resemblance in their properties to the antibiotic subtilin.

We wish to record our thanks to Mr J. Barrett for assistance in assaying samples of nisin and to $\mathrm{Mr}$ Omar Boys whose help has been invaluable in running the paper chromatograms. We are grateful to Bengers Ltd. for gifts of nisin and to Mr S. Wijesundera and Mr F. Gibson of the Department of Microbiology, Oxford, for a gift of cystathionine.

One of us (G.G.F.N.) is grateful to the Medical Research Council for a part-time personal grant. We are indebted to the Medical Research Council for grants for technical assistance and materials. 


\title{
REFERENCES
}

Bavin, E. M., Beach, A. S., Falconer, R. \& Friedmann, R. (1952). Lancet, i, 127.

Berridge, N. J. (1949). Biochem. J. 45, 486.

Berridge, N. J. (1952). Nature, Lond. (in the Press).

Berridge, N. J. \& Barrett, J. (1951). Nature, Lond., 167, 448.

Callow, R. K. \& Work, T. S. (1952). Personal communication and Biochem. J. 51, 558.

Campbell, W. R. \& Hanna, M. J. (1937). J.biol. chem. 119, 1.

Consder, R., Gordon, A. H. \& Martin, A. J. P. (1946). Biochem. J. 40, 580.

Craig, L. C. \& Craig, D. (1950). Technique of Organic Chemistry, 3, 200. New York: Interscience.

Craig, L. C., Gregory, J. D. \& Barry, G. T. (1949). Cold Spr. Harb. Symp. quant. Biol. 14, 24.

Craig, L. C., Hausmann, W., Ahrens, E. H. \& Harfenist, E. J. (1951). Anal. Chem. 23, 1236.

Craig, L. C. \& Post, 0. (1949). Anal. Chem. 21, 500.

Dent, C. E. (1948). Biochem. J. 43, 169.

Dimick, K. P., Alderton, G., Lewis, J. C., Lightbody, H. D. \& Fevold, H. C. (1947). Arch. Biochem. 15, 1.

Gowans, J. L., Smith, N. \& Florey, H. W. (1952). Brit. J. Pharmacol. (in the Press).
Hirsch, A. (1951). Nature, Lond., 167, 1031.

Horn, M. J., Jones, D. B. \& Ringel, S. J. (1941). J. biol. Chem. 138, 141.

Horowitz, N. H. (1947). J. biol. Chem. 171, 255.

Küster, W. \& Irion, W. (1929). Hoppe-Seyl. Z. 184, 225.

Lewis, J. C. \& Alderton, G. (1948). Abstr. Amer. chem. Soc. 114th Meeting, 61-62 C.

Mattick, A. T. R. \& Hirsch, A. (1944). Nature, Lond., 154, 551.

Mattick, A. T. R. \& Hirsch, A. (1947). Lancet, ii, 5.

Moore, S. \& Stein, W. H. (1948). J. biol. Chem. 176, 367.

Mozingo, R., Wolf, D. E., Harris, S. A. \& Folkers, K. (1943). J. Amer. chem. Soc. 65, 1013.

Newton, G. G. F. \& Abraham, E. P. (1950). Biochem. J. 47, 257.

Newton, G. G. F. \& Abraham, E. P. (1952). Unpublished observations.

Sanger, F. \& Tuppy, H. (1951). Biochem. J. 49, 463.

Tompkins, E. R. \& Kirk, P. L. (1942). J. biol. Chem. 142, 477.

Williamson, B. \& Craig, L. C. (1947). J. biol. Chem.168, 687. Woiwod, A. J. (1949). J. gen. Microbiol. 3, 312.

\section{Studies in Vitamin A \\ 19. PREPARATION AND PROPERTIES OF RETINENE}

\author{
By H. R. CAMA, P. D. DALVI, R. A. MORTON, M. K. SALAH, \\ G. R. STEINBERG AND A. L. STUBBS \\ Department of Biochemistry, University of Liverpool
}

\section{(Received 9 February 1952)}

Retinene, $_{2}$ was first recognized by Wald (1937) and described as a 'deep yellow carotenoid' obtained from the retinas of freshwater fishes; when dissolved in chloroform it showed a wide absorption band with $\lambda_{\max }$ at $405 \mathrm{~m} \mu$., and in the presence of excess of the antimony trichloride reagent it gave a greenish blue colour with $\lambda_{\max }$ at 702-706 $\mathrm{m} \mu$.

The retinas of freshwater fishes are violet in colour (Kühne \& Sewall, 1880), whereas those of most animals and sea fishes are pink. The spectral shift (Köttgen \& Abelsdorff, 1896) is now ascribed (Wald, 1937, 1938-9a,b) to the partial or complete replacement of visual purple (rhodopsin) by porphyropsin with an absorption peak near $522 \mathrm{~m} \mu$. instead of $500 \mathrm{~m} \mu$.

Exposure to light liberates retinene $e_{1}$ from rhodopsin and retinene $e_{2}$ from porphyropsin, but the amounts obtainable from retinas are of course too small for other than spectroscopic identification. Retinene ${ }_{1}$ has been shown to be vitamin $A_{1}$ aldehyde and can now be obtained in quantity by oxidation of vitamin $A_{1}$ alcohol (Ball, Goodwin \& Morton, 1948). Similarly, retinene $e_{2}$ is the aldehyde of vitamin $\mathbf{A}_{2}$ (Morton, Salah \& Stubbs, 1947), but its preparation in a state of purity has been difficult.
The term vitamin $\mathbf{A}_{\mathbf{2}}$ was first used by Edisbury, Morton \& Simpkins (1937) on the strength of the very close analogy between the distribution of vitamin $A_{1}$ in the organs of marine fishes and the distribution of a ' $693 \mathrm{~m} \mu$. chromogen' in freshwater fishes. The antimony trichloride colour test applied to vitamin $A_{1}$ gives a blue solution ( $\lambda_{\max }$ $620 \mathrm{~m} \mu$.), whereas when applied to vitamin $A_{2}$ it gives a greenish blue solution $\left(\lambda_{\max } 693 \mathrm{~m} \mu\right.$.). The ultraviolet absorption peaks are at 325-328 $\mathrm{m} \mu$. for vitamin $A_{1}$ and $350-352,286 \mathrm{~m} \mu$. for vitamin $A_{2}$. The $693 \mathrm{~m} \mu$. chromogen had been described earlier (Heilbron, Gillam \& Morton, 1931). Its importance was clearly recognized by Lederer \& Rosanova (1937) and Wald (1938-9a).

The problems to be discussed in this and succeeding papers have a rather complicated background which needs explanation.

(1) Fish-liver oils with a high vitamin $A_{2}$ content are not easy to obtain; most commercial oils are of marine origin and vitamin $A_{2}$, although frequently present, rarely accounts for more than 5-10\% of the vitamin A content. For the British freshwater fishes which have been studied, the absolute amount of vitamins A present in liver oils is quite low and the 